\title{
Sacroiliac tuberculosis masquerading as mechanical lower back pain in a collegiate basketball athlete: a case presentation
}

\author{
M Moyaert,1,3,4 MD, M Med (Sports Med); J T Viljoen,1, 2, $\mathbf{3}$ BSc (Physio), MPhil (Exercise Sci); P L Viviers, 1, 2, ${ }^{3}$ MBChB, M Med \\ Sc, MSc (Sports Med), FACSM; W Derman, ${ }^{1}{ }^{2}$ MBChB, BSc (Med) (Hon), PhD, FFIMS; R de Villiers, ${ }^{\mathbf{5}}$ MBChB; MMed (Rad. D) \\ ${ }^{1}$ Institute of Sport and Exercise Medicine, Division of Orthopaedics, Faculty of Medicine and Health Sciences, Stellenbosch University, South Africa \\ ${ }^{2}$ IOC Research Centre, Cape Town, South Africa \\ ${ }^{3}$ Campus Health Service, Stellenbosch University, South Africa \\ ${ }^{4}$ Catholic University of Leuven, Department of Physical Medicine and Rehabilitation, Leuven, Belgium \\ ${ }^{5}$ Winelands Radiology, Institute of Orthopaedics and Rheumatology, Stellenbosch, South Africa
}

Corresponding author: P L Viviers (plviviers@sun.ac.za)

Background: Sacroiliac tuberculosis is a rare condition for which early diagnosis and effective management frequently proves challenging. This report describes a case that was initially overlooked due to its presentation and unreported constitutional symptoms.

Aim: To alert clinicians about skeletal tuberculosis, an often neglected diagnostic differential, which requires a high index of clinical suspicion, especially for patients from endemic areas.

Findings: This patient's presentation (sports injury) and unreported constitutional symptoms resulted in a delay in the diagnosis and initial institution of treatment.

Implications: This report illustrates the importance of specifically asking about constitutional symptoms, even in sports injury settings and being mindful of infectious diseases or other chronic medical conditions, which may masquerade as common sports injuries.

Keywords: skeletal tuberculosis, sacroiliitis, hip pain

S Afr J Sports Med 2018; 30:1-3. DOI: 10.17159/2078-516X/2018/v30i1a4372

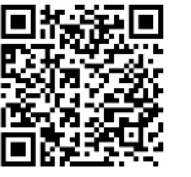

Tuberculosis (TB) is a major cause of death worldwide. In Sub-Saharan Africa, the disease burden is especially high with South Africa being one of the top seven global countries with the highest estimated numbers of TB cases (454 000 per year).[1] Skeletal TB accounts for 10\% of the extrapulmonary manifestations, of which only $5-8 \%$ affect the sacroiliac joint.[2]

Diagnosis of sacroiliac TB often proves challenging due to its insidious onset, non-specific clinical picture and low index of suspicion.[3] The most frequently reported clinical symptoms include persistent lower back pain and antalgic gait, [2-6] while several authors also report a positive straight leg raise test. $[3,4]$ This is likely due to the close anatomical relationship between the sacroiliac (SI) joint, hip, lumbosacral plexus and the intrapelvic space.

Despite previous efforts to describe the symptomology and clinical presentation of sacroiliac TB, no specific mention is given to its manifestation in athletes.[3-5] This requires further elucidation, particularly as a consequence of misdiagnosis or a delay in initiation of treatment when eventual complications, such as sacroiliac joint destruction, formation of a local abscess, vertebral collapse or neurological fallout, may arise.[3,5] All of these are potentially detrimental to the athlete's general health, well-being and performance.

\section{Case report}

A 21-year-old male collegiate basketball player of SubSaharan African origin presented to the sports injury clinic at
Stellenbosch University with a four week history of spontaneous onset, progressive right-sided lumbosacral pain and stiffness. No history of trauma or any other inciting event was reported. Further medical history was unremarkable, except for episodes of intermittent fatigue experienced over the past three years, related to a previously diagnosed chronic iron deficiency anaemia. At the time of this evaluation, an extensive laboratory blood workup, including a full blood count, platelet count, measures of erythrocyte sedimentation rate, as well as C-reactive protein and ferritin concentrations, were conducted. These tests revealed low ferritin concentrations, mean corpuscular volume and red cell haemoglobin concentrations. Treatment ensued consisting of iron (ferrous sulphate) and multivitamin supplementation; however, poor compliance and failure to return for follow-up assessments hindered resolution of this patient's problem.

Physical examination revealed palpation tenderness of the right SI joint. The FABER test of the right hip, straight leg raise test and Gaenslen's test were positive. In comparison, the left side was normal. No neurological abnormalities could be found related to either the lumbar spine or lower extremities. Plain radiographs of the pelvis were reported as normal. A provisional diagnosis of mechanical sacroiliac joint dysfunction was made and anti-inflammatory medication was prescribed for pain relief. The patient was referred for physiotherapy treatment (including soft tissue mobilisation, hip stability exercises, dry needling and other supportive modalities).

Due to a poor response to conservative treatment and a 

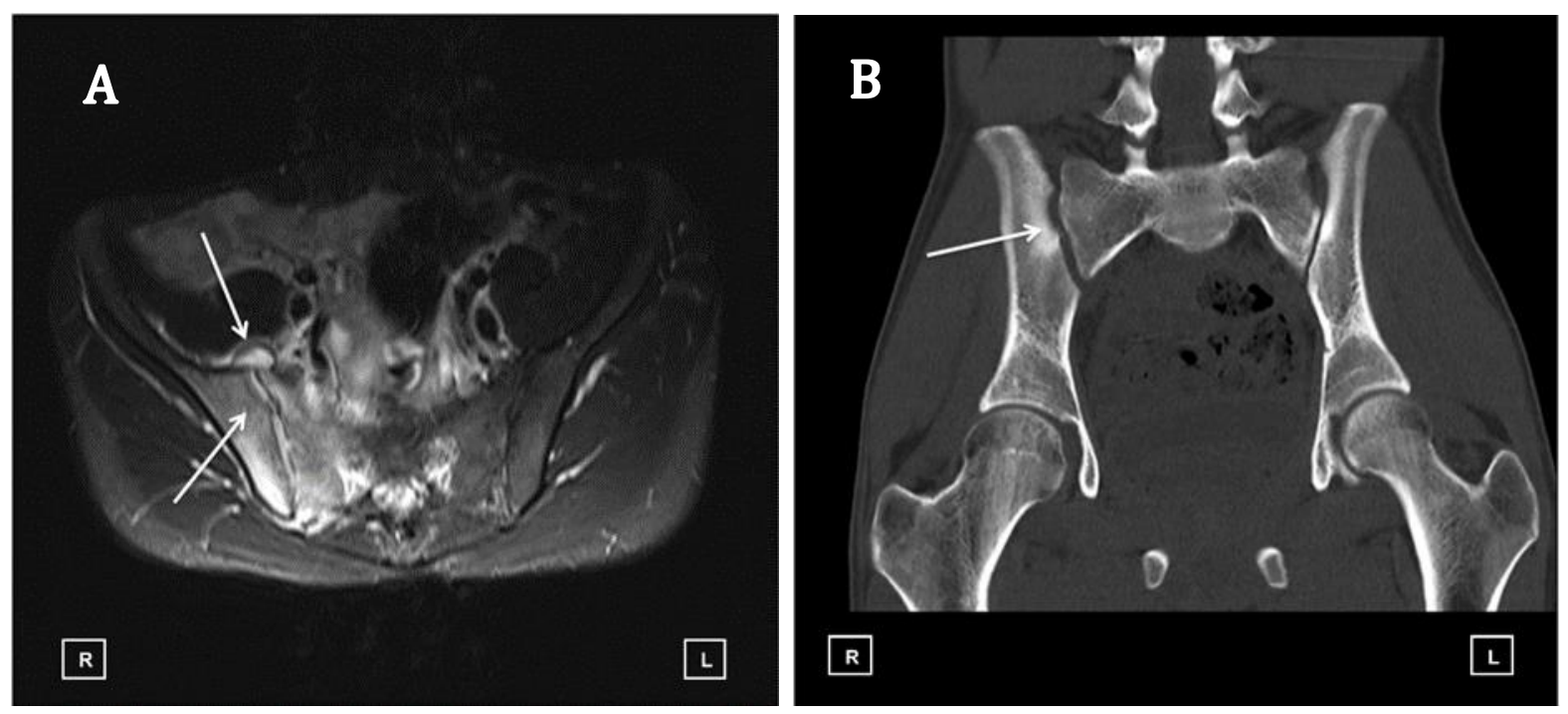

Fig.1A. MRI of pelvis: T1-fat saturation post-contrast. Enhancing SI-joint with bone marrow oedema of the sacrum and right iliac bone. SI joint fluid collection with anterior extension deep to right iliacus muscle. Features compatible with an infective sacroiliitis.

Fig.1B. Coronal CT of pelvis showing widening of the right SI joint with erosion and sclerosis of the right ilium. Normal left SI joint.

noticeable deterioration in his gait pattern, the patient was reassessed after eight weeks. At the time, a retrospect enquiry revealed further constitutional symptoms, which were not mentioned in the initial history, including weight loss (from $61 \mathrm{~kg}$ to $54 \mathrm{~kg}$ over a period of two months), intermittent night sweating, fatigue and a transient cough. Further investigations were deemed necessary, including blood laboratory tests, which showed elevated CRP (C - reactive protein) concentrations of $123.8 \mathrm{mg} / \mathrm{L}$ (normal reference $<5.0 \mathrm{mg} / \mathrm{L}$ ), an increased ESR (erythrocyte sedimentation rate) of $75 \mathrm{~mm} / \mathrm{hr}$ (normal reference $2-28 \mathrm{~mm} / \mathrm{hr}$ ) and a blood count within the normal range. Tests for the presence of human leukocyte antigen (HLA-B27) and human immunodeficiency virus (HIV) were also performed to exclude other possible causes, such as immune-mediated diseases. However, these proved to be negative.

Magnetic resonance imaging (MRI) and computed tomography $(\mathrm{CT})$ of the pelvis revealed an effusion of the right SI joint with extensive oedema in the bone marrow and adjacent muscles (Figs. 1A and B). These images were highly suggestive of right-sided sacroiliitis with features of an infective process. At this point, an additional differential diagnosis of tuberculosis (TB) was considered as the patient originates from a TB endemic region in Sub-Saharan Africa and had frequent contact with a relative who had TB. Diagnosis was later confirmed using a needle core biopsy of the sacrum, together with subsequent PCR (polymerase chain reaction) analysis, which was positive for Mycobacterium tuberculosis (GeneXpert PCR). Sensitivity for rifampicin was established and multidrug resistance was excluded. The patient was initiated on a four-drug combination therapy (isoniazid, rifampicin, pyrazinamide and ethambutol) with additional Vitamin B6 (pyridoxine) supplementation for a minimum duration of 9-12 months. Continuous monitoring of the patient consisted of clinical re-evaluation supported by standard radiographs and serial ESR/CRP counts every six weeks for the first three months and then every three months for the duration of the treatment.

After receiving almost four months of anti-tuberculosis treatment, the patient showed a good clinical response. Follow-up blood counts revealed a reduction in ESR (75 $\mathrm{mm} / \mathrm{Hr}$ vs. $2 \mathrm{~mm} / \mathrm{Hr}$ ) and CRP (123.8mg/L vs. $3.1 \mathrm{mg} / \mathrm{L})$, while radiography showed no progression compared to previous findings. The patient has since commenced a physical rehabilitation programme that includes progressive strengthening and aerobic conditioning.

\section{Discussion}

In this case, the patient's initial presentation with a "sports injury" and unreported constitutional symptoms resulted in a differential diagnosis, including sciatica, gluteal muscle strain and infectious/inflammatory disorders of the SI joint. This led to a final working diagnosis of sacroilitis, followed by referral for appropriate physiotherapy treatment. However, the patient's failure to respond to the prescribed treatment alerted the authors to the possibility of a missed diagnosis. Consequently, a repeat clinical assessment, including an indepth history taking, physical examination and special tests, resulted in the identification of underlying sacroiliac TB. This demonstrates the importance of an accurate clinical assessment in order to identify underlying chronic disease in the presence of a sports injury. Furthermore, a high index of clinical suspicion should be maintained for chronic medical conditions as this can influence the initial differential as well as the final working diagnosis.

Plain radiography (X-ray) is one of the various screening methods used to assist clinicians with an effective diagnosis of musculoskeletal TB. In this case, subtle features suggestive of joint pathology were indeed visible retrospectively on initial standard anterior - posterior (AP) radiographs of the pelvis; however, these were not recognised at the time. Subsequent MRI and CT confirmed features of sacroilitis stemming from an infective origin. Together with the history of constitutional 
symptoms for TB further investigation was warranted using specific blood markers and core needle biopsy. It is important to note that exclusive diagnosis of $\mathrm{TB}$ is typically demonstrated by either acid-fast bacilli (AFB) on microscopy, growth of Bacilli in a Lowestein-Jensen culture or identification of granulomatous lesions in the histologic specimen from an open biopsy or fine needle aspiration. ${ }^{[4,6]}$ Considering that the traditional techniques using cultures can be time-consuming, two polymerase chain reaction methods are frequently used, namely the Line Probe Assay (LPA) method or the GeneXpert PCR (GXP) method. The latter method was used to confirm diagnosis of sacroiliac TB in this case.

\section{Conclusion}

This case demonstrates important messages for the clinician working in sport and exercise medicine. Firstly, chronic medical conditions can masquerade as common sports injuries, and conversely, exercise or sport may often reveal the chronic illness. It is therefore important that the clinician takes a meticulous medical history and is mindful of a wide differential during an assessment of common sports injuries, particularly when no trauma or inciting event is involved. The second important lesson is that patients should be completely reassessed should they not respond as anticipated or according to the treatment plan set out for the particular patient.
Patient consent: We would like to thank the patient for his consent to publish his clinical information.

Study funding and conflict of interest: The authors report no funding or conflict of interest.

\section{References}

1. Global tuberculosis report 2017. Geneva: World Health Organization.

http://www.who.int/tb/publications/global_report/en/

2. Peto HM, Pratt RH, Harrington TA, et al. Epidemiology of extrapulmonary tuberculosis in the United States, 1993-2006. Clin Infect Dis 2009;49(9):1350-1357.[doi:10.1086/605559]

3. Prakash J. Sacroiliac tuberculosis - A neglected differential in refractory low back pain - Our series of 35 patients. J Clin Orthop Trauma 2014;5(3):146-153. [doi:10.1016/j.jcot.2014.07.008]

4. Gao F, Kong XH, Tong XY, et al. Tuberculous sacroiliitis: a study of the diagnosis, therapy and medium-term results of 15 Cases. J Int Med Res 2011;39(1):321-335. [doi:10.1177/147323001103900135]

5. Gelal F, Sabah D, Doğan R, et al. Multifocal skeletal tuberculosis involving the lumbar spine and a sacroiliac joint: MR imaging findings. Diagn Interv Radiol 2006;12(3):139-141. [PMID: 16972219]

6. Patwardhan SA, Joshi S. Laboratory diagnosis of spinal tuberculosis: Past and present. ArgoSpine News J 2011;23(3):120-124.[doi:10.1007/s12240-011-0023-9] 